\title{
Effect of pilocarpine mouthwash on salivary flow
}

R. Bernardi, C. Perin, F.L. Becker, G.Z. Ramos, G.Z. Gheno, L.R. Lopes, M. Pires and H.M.T. Barros

\author{
Divisão de Farmacologia Básica e Clínica, \\ Departamento de Ciências Fisiológicas, \\ Fundação Faculdade Federal de Ciências M édicas \\ de Porto Alegre, Porto Alegre, RS, Brasil
}

\section{Correspondence \\ H.M.T. Barros \\ Divisão de Farmacologia, FFFCMPA \\ Rua Sarmento Leite, 245 \\ 90050-170 Porto Alegre, RS \\ Brasil \\ Fax: + 55-51-224-8822 \\ E-mail: helenbar@ fffcmpa.tche.br}

Received January 2, 2001

Accepted September 27, 2001

\section{Abstract}

Pilocarpine is a cholinergic agonist that increases salivary flow and has been used to treat xerostomia. Oral intake is the most frequent route of administration. Adverse effects are dose-dependent and include sudoresis, facial blushing and increased urinary frequency. The objective of the present study was to evaluate the effects of topical pilocarpine solutions as mouthwashes on salivary flow and their adverse effects on healthy subjects. Forty volunteers received $10 \mathrm{ml}$ $0.5,1$ and $2 \%$ pilocarpine solutions or $0.9 \%$ saline in a randomized, double-blind, placebo-controlled manner. Salivation was measured before and 45, 60 and $75 \mathrm{~min}$ after mouth rinsing for $1 \mathrm{~min}$ with $10 \mathrm{ml}$ of saline or pilocarpine solutions. Vital signs were measured and ocular, gastrointestinal and cardiovascular symptoms, anxiety and flushing were estimated using visual analog scales. There was a dosedependent increase in salivation. Salivation measured after 1 and $2 \%$ pilocarpine ( $1.4 \pm 0.36$ and $2.22 \pm 0.42 \mathrm{~g}$, respectively) was significantly $(\mathrm{P}<0.001)$ higher than before $(0.70 \pm 0.15$ and $0.64 \pm 0.1 \mathrm{~g})$, with a plateau between 45 and $75 \mathrm{~min}$. Cardiovascular, visual, gastrointestinal and behavioral symptoms and signs were not changed by topical pilocarpine. Mouth rinsing with pilocarpine solutions at concentrations of 1 to $2 \%$ induced a significant objective and subjective dose-dependent increase in salivary flow, similar to the results reported by others studying the effect of oral $5 \mathrm{mg}$ pilocarpine. The present study revealed the efficacy of pilocarpine mouthwash solutions in increasing salivary flow in healthy volunteers, with no adverse effects. Additional studies on patients with xerostomia are needed.

\section{Introduction}

Dry mouth or xerostomia is a common symptom most frequently associated with salivary gland hypofunction induced by transitory physiologic states, pathologic conditions or as a side effect of drug therapy or radiation (1-3). Xerostomia almost always develops after salivary gland destruction in

\author{
Key words \\ - Pilocarpine \\ - Mouth rinsing \\ - Mouthwash \\ - Xerostomia \\ - Salivation
}


other factor that may induce salivary gland hypofunction because of chemical salivary changes, the use of medications or associated systemic diseases $(10,17)$.

Chronic xerostomia compromises chewing, swallowing and speaking and predisposes to oral cavities and to a variety of destructive processes that include thinning, atrophy and ulceration of mucosa and fungal infections. All of these symptoms may be responsible for nutritional deficiencies and difficulties in communication and sleeping, leading to an overall decline in quality of life $(2,5,7)$.

The treatment goals of patients with dry mouth are to relieve symptoms, to prevent or correct the consequences of salivary dysfunction, and to treat underlying disease (3). A number of treatments have been proposed to improve salivary function. Symptomatic treatments with saliva replacement (artificial saliva), hydration of oral mucosa with small sips of water, mastication or topical gustatory stimulation are largely palliative and generally offer only short-term benefits $(6,18,19)$. Therefore, preventive therapies with fluoride, remineralizing solutions and antifungals may be necessary (3). Numerous systemic medications such as bromhexine, anetholetrithione and pilocarpine hydrochloride (3) have been tested as salivary stimulants. The most extensively studied sialagogue agent is pilocarpine (6).

Pilocarpine is a parasympathomimetic drug that exhibits potent muscarinic-stimulating properties. The pharmacological effects of this agent are multiple and include increase of smooth muscle tone and motility of the gastrointestinal and urinary tracts, gallbladder, biliary ducts and bronchi. An outstanding enhancement of salivary and sweating gland secretion is observed with pilocarpine (20) and these properties have been known in Western medicine for more than 100 years $(20,21)$. Controlled studies to investigate the ability of pilocarpine to stimulate salivary flow and to relieve oral dryness symptoms initiated in the 1960s. The results showed that pilocarpine is superior to placebo to relieve xerostomia complaints. At the present time it has been well established that oral pilocarpine is able to improve sicca symptoms, in particular those related to eyes and mouth in patients with systemic disorders such as Sjögren's syndrome (22-24) or other autoimmune conditions (25) and secondary to radiation therapy (26). After oral administration, the most common side effect is sweating, but urinary and gastrointestinal disturbances are also frequently reported $(3,6,22)$. On the other hand, because pilocarpine is a parasympathomimetic drug, there is some risk of cardiovascular and pulmonary effects that make systemic administration somewhat dangerous. Patients with gastric ulcer and uncontrolled asthma should not use pilocarpine. Likewise, this drug must be used carefully by patients with controlled asthma, chronic bronchitis, pulmonary or cardiac disease, systemic hypertension, or using B-adrenergic blockers $(6,7)$. Thus, a systematic approach to the study of the efficacy and potential side effects of topical pilocarpine administration used to enhance salivary flow is essential because of the possibility to use smaller dosages that could show a direct local effect with minimal systemic manifestations.

The purpose of the present study was to investigate the effect of pilocarpine solutions at different concentrations, applied by mouth rinsing, on salivary flow of healthy volunteers as well as to determine possible side effects secondary to this route of administration.

\section{Subjects and Methods}

The study was a placebo-controlled, double-blind, randomized clinical trial approved by the Research Ethics Committee. The sample was composed of 40 healthy volunteers, medical students from the Fundação Faculdade Federal de Ciências 
Médicas, Porto Alegre, RS, Brazil, 18 to 30 years old, of both sexes (26 females and 14 males). Exclusion criteria were bronchial asthma, history of hypertension or hypotension, recent use of drugs, including smoking, suspected or confirmed pregnancy, heart, renal or hepatic diseases, peptic ulcer, hyperthyroidism, epilepsy, Parkinson's disease or parkinsonian syndrome, and HIV infection. Individuals who had experienced chemical or mechanical salivary stimulation (eating, drinking, smoking, oral hygiene, or chewing gum) within 90 min before the experiment were also excluded. To participate as volunteers all individuals signed a written informed consent term. After selection, the volunteers were randomly allocated to four groups, each consisting of 10 individuals homogeneously distributed by age and sex, in accordance with the solution to be used: $0.5,1$ or $2 \%$ pilocarpine or $0.9 \%$ saline.

Before the administration of solutions, each volunteer completed an analog scale (4) consisting of 11 elements to determine the intensity of symptoms of anxiety, tremor, sudoresis, facial flushing, abdominal and/or thoracic distress, lacrimation, salivation, palpitation, nausea, visual disturbances, and hunger. The individual could indicate a point on a $10-\mathrm{cm}$ long line delimitated by words expressing the highest and the lowest degree of each symptom. Blood pressure and heart rate were measured with a portable wrist blood pressure monitor (Omron, Vernon Hill, IL, USA). Salivary flow was quantified by weighing a piece of cotton $(4 \times 1 \mathrm{~cm})$ that was kept under the tongue for $1 \mathrm{~min}$. Salivary flow was measured before and 45, 60 and 75 min after mouth rinsing.

After the first salivary flow measurement, the volunteers were instructed to maintain $10 \mathrm{ml}$ of the solution in the mouth for 1 min, without swallowing the solution and being very careful to spit the entire volume after this time. By the end of the 75-min period, blood pressure and heart rate were measured again, the volunteers were in- structed to respond to the same analog scale used at the beginning of the experiment and were asked to estimate which solution had been assigned to them.

Statistical analysis was performed with the Sigma Stat 2.0 software (Jandel Scientific, Chicago, IL, USA) using a two-way repeated measures ANOVA for time and pilocarpine dose. Linear regression was used to establish the dose-response curve for pilocarpine solutions with respect to salivary flow. The replies to the analog scales were analyzed by one-way ANOVA. Post hoc dose comparisons were made by the Student-Newman-Keuls test. Multiple linear regression was used to verify the correlation between adverse effect parameters taken from analog scales and salivary flow. The Fisher exact test was used to compare the differences between treatment groups to correctly estimate the solution received. Statistical significance was defined as $\mathrm{P}<0.05$.

\section{Results}

A post hoc comparison between groups indicated that mouth washing with 1 or $2 \%$ pilocarpine solutions significantly $(\mathrm{F}=4.803$, $\mathrm{P}=0.006)$ increased salivary flow compared to saline solution. There was no significant difference in salivation at 45, 60 and $75 \mathrm{~min}$ after mouth rinsing with pilocarpine solutions $(\mathrm{F}=1.050, \mathrm{P}=0.382)$. The increase in salivary flow induced by 1 and $2 \%$ pilocarpine was stable along the three moments of quantification, as illustrated in Figure 1. There was a dose-related increase in salivary flow (linear regression equation: $\mathrm{y}=0.601+$ $0.818 \mathrm{x} ; \mathrm{SE}=1.042 ; \mathrm{R}=0.512$ ).

The $2 \%$ pilocarpine solution was associated with increased sensation of salivary flow (sialorrhea), while the other pilocarpine concentrations and saline solution did not modify individual perception of salivation. All individuals who had received $2 \%$ pilocarpine or saline solution guessed correctly which solution had been administered. The 
Figure 1. Salivary flow $(\mathrm{g} / \mathrm{min})$ prior (Pre) to pilocarpine treatment and 45, 60 and 75 min after mouth washing with $10 \mathrm{ml}$ of $0.5,1$ or $2 \%$ pilocarpine solutions or saline as the control solution for $1 \mathrm{~min}$. *P<0.05 compared to control (Student-Newman -Keuls test). rates of correct answers to this same question were $80 \%$ for $1 \%$ pilocarpine and $50 \%$ for $0.5 \%$ pilocarpine.

No significant effects were observed in heart rate or blood pressure $90 \mathrm{~min}$ after administration of the pilocarpine solutions. Likewise, all other symptoms investigated did not differ significantly between groups (Table 1). However, the multiple linear regression test showed moderate but significant correlation $\left(\mathrm{R}=0.72, \mathrm{~F}_{5,34}=7.338\right)$ between the salivation parameter and symptoms of sialorrhea $(\mathrm{P}=0.04)$, systolic pressure $(\mathrm{P}=0.005)$, facial flushing $(\mathrm{P}=0.005)$, thoracic distress $(\mathrm{P}=0.011)$, and palpitations $(\mathrm{P}=0.020)$.

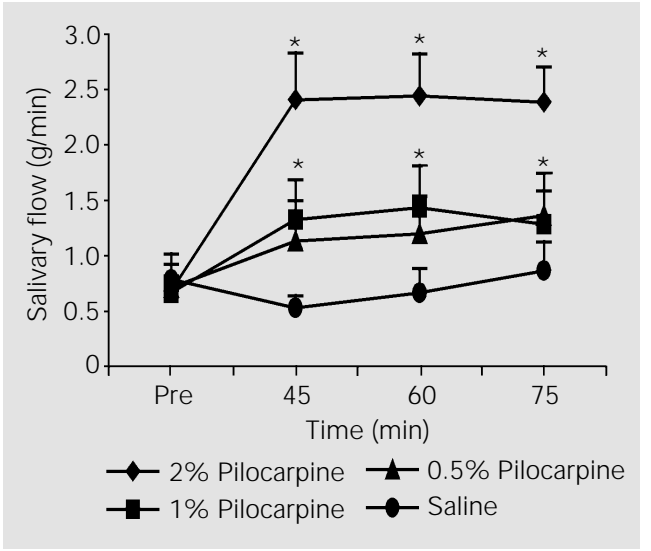

\section{Discussion}

Regardless of the causes of dry mouth or xerostomia (secondary to autoimmune disease, radiation, medications or aging) this condition induces several limitations to patients besides those produced by the primary disease. One has to keep in mind that these facts, combined with chronic diseases and/ or treatments, make it necessary to search for a safe therapy of xerostomia.

Nowadays, 5 to $10 \mathrm{mg}$ pilocarpine given by the oral route three or four times daily is considered to be safe and effective in stimulating salivary flow in patients with mild to severe secretory hypofunction (3). The mechanisms responsible for the effects of pilocarpine on salivary flow involve local and direct cellular stimulation (18). The parasympathetic action of pilocarpine induces water and electrolyte flow in saliva. There is evidence that pilocarpine also stimulates the production of mucin and of several other salivary constituents (4,19,27-29). Mucins are important to prevent infections and chemical or mechanical traumas to oral structures. Because mucin production occurs mostly in the small salivary glands, any increase in mucin will reduce xerostomia. Consequently,

Table 1. Estimation of adverse effects on healthy volunteers after mouth rinsing with pilocarpine solutions using visual analog scales.

\begin{tabular}{lccccr}
\hline Symptom & Saline & Pilocarpine (0.5\%) & Pilocarpine (1\%) & Pilocarpine (2\%) & $F_{3,36}$ \\
\hline Sialorrhea & $6.0 \pm 0.65$ & $5.7 \pm 0.81$ & $6.6 \pm 0.63$ & $8.9 \pm 0.5^{*}$ & 4.803 \\
Nervousness & $1.9 \pm 0.58$ & $2.0 \pm 0.84$ & $3.3 \pm 1.15$ & $2.6 \pm 0.71$ & 0.574 \\
Tremor & $1.7 \pm 0.66$ & $1.6 \pm 0.54$ & $2.0 \pm 0.86$ & $2.5 \pm 0.98$ & 0.266 \\
Sweating & $1.7 \pm 1.01$ & $4.3 \pm 1.29$ & $4.9 \pm 1.34$ & $4.4 \pm 1.32$ & 1.326 \\
Facial flushing & $1.3 \pm 0.36$ & $2.9 \pm 1.06$ & $4.3 \pm 1.31$ & $2.5 \pm 1.10$ & 1.482 \\
Abdominal distress & $1.9 \pm 1.04$ & $2.8 \pm 1.23$ & $0.7 \pm 0.39$ & $1.7 \pm 1.0$ & 0.785 \\
Lacrimation & $0.8 \pm 0.35$ & $0.4 \pm 0.51$ & $0.7 \pm 0.59$ & $0.3 \pm 0.21$ & 0.749 \\
Palpitations & $1.7 \pm 0.68$ & $1.7 \pm 0.71$ & $0.9 \pm 0.58$ & $0.6 \pm 0.26$ & 0.906 \\
Nausea & $0.4 \pm 0.16$ & $1.3 \pm 0.77$ & $1.0 \pm 0.68$ & $0.8 \pm 0.32$ & 0.475 \\
Thoracic distress & $0.6 \pm 0.34$ & $0.4 \pm 0.16$ & $0.4 \pm 0.16$ & $0.5 \pm 0.22$ & 0.168 \\
Blurred vision & $0.4 \pm 0.16$ & $1.1 \pm 0.58$ & $2.3 \pm 1.02$ & $1.5 \pm 0.99$ & 1.049
\end{tabular}

Data are reported as means $\pm S E M$. Bold represents significant $F$ values (ANOVA $=P<0.05$ ).

$* \mathrm{P}<0.05$ compared to control (Student-Newman-Keuls test). 
even a small increase in salivary flow may induce symptomatic improvement for those patients who are treated with oral pilocarpine (3-5). On the other hand, several weeks must elapse before the symptomatic improvement becomes apparent because the reversal of the atrophic and desiccated changes induced by salivary deprivation does not occur immediately after pilocarpine (3-5). The longterm benefits of oral pilocarpine treatment against the consequences of xerostomia such as dental cavities or oral candidiasis, which are already experimentally demonstrated $(15,30)$, require further studies in humans $(6,31,32)$. There is no indication of the development of tolerance to the stimulant effect of pilocarpine on salivary flow $(3,6)$.

In the present study, mouth rinsing with 1 and $2 \%$ pilocarpine solutions was able to induce a significant and dose-dependent elevation in salivary flow perceived subjectively and objectively, similar to what happens when pilocarpine is administered by the oral route $(2,4,6,21,33,34)$. The latency to increase salivation when pilocarpine is administered by the oral route is $15 \mathrm{~min}$, with a peak at $60 \mathrm{~min}$ and a duration of 2 or $3 \mathrm{~h}(2,6,27)$. Even though it is not possible to compare the latency of salivation after oral or topical pilocarpine administration it was clear that after topical administration the peak effect must have occurred earlier than 1 $\mathrm{h}$ and was stable for at least $75 \mathrm{~min}$.

The adverse effects of pilocarpine are exaggerate responses of the autonomic nervous system. Some of these adverse effects are frequently reported after chronic use of oral pilocarpine and are dose-dependent $(4,5)$. Sudoresis (37 to $65 \%$ ), increased urinary frequency (38\%) and facial flushing (12 to $42 \%)$ are most likely to occur $(4,6,18,27)$. Topical use of pilocarpine by mouth rinsing at the highest concentration used (2\%) did not modify the blood pressure level or heart rate of healthy volunteers, comparable to when this drug is used orally $(2,4,6,27)$. On the other hand, it was determined that facial flushing, palpitations and thoracic distress were positively correlated with the sialagogue effect. Since the linear regressions were significant, it is possible to infer that if excessive salivation is produced with higher pilocarpine doses than the ones used here, increased systolic pressure, facial flushing, thoracic distress and palpitations are the first adverse effects to be expected. In addition, the demonstration that salivary flow could be anticipated by the perception of increased salivation demonstrates a positive correlation between objective and subjective parameters. This finding also indicates that pilocarpine is effective in enhancing salivation and relieving xerostomia.

This clinical phase I study demonstrates that mouth washing with $10 \mathrm{ml} 1$ and $2 \%$ pilocarpine solutions increases salivary flow in healthy volunteers without inducing harmful adverse effects. More studies will be necessary to determine the efficacy of pilocarpine through this route of administration for treatment of xerostomic patients.

\section{References}

1. Butt GM (1991). Drug-induced xerostomia. J ournal of the Canadian Dental Association, 57: 391-393.

2. Fox PC, Atkinson J C, Macynski AA, Wolff A, Kung DS, Valdez IH, Jackson W, Delapenha RA, Shiroky J \& Baum BJ (1991). Pilocarpine treatment of salivary gland hypofunction and dry mouth (xerostomia). Archives of Internal Medicine, 151: 1149-1152.
3. Fox PC (1997). Management of dry mouth. Dental Clinics of North America, 41: 863-875.

4. LeVeque FG, Montgomery M, Potter D, Zimmer MB, Rieke JW, Steiger BW, Gallagher SC \& Muscoplat CC (1993). A multicenter, randomized, double-blind, placebo-controlled, dose-titration study of oral pilocarpine for treatment of radiationinduced xerostomia in head and neck can- cer patients. J ournal of Clinical Oncology, 11: 1124-1131.

5. J ohnson J T, Ferretti GA, Nethery J, Valdez $\mathrm{IH}$, Fox PC, Ng D, Muscoplat CC \& Gallagher SC (1993). Oral pilocarpine for post-irradiation xerostomia in patients with head and neck cancer. New England J ournal of Medicine, 329: 390-395.

6. Wiseman LR \& Faulds D (1995). Oral pilocarpine: A review of its pharmacological 
properties and clinical potential in xerostomia. Drugs, 49: 143-155.

7. Oxholm P, Prause J U \& Schiødt M (1998). Rational drug therapy recommendations for the treatment of patients with Sjögren's syndrome. Drugs, 56: 345-353.

8. Marracini RL, Reynolds 3rd CF, Houck PR, Miller MD, Frank E, Perel J M, Cornes C, Mazumdar S \& Kupfer DJ (1999). A double-blind, placebo-controlled assessment of nortriptylin's side-effects during 3-year maintenance treatment in elderly patients with recurrent major depression. International J oumal of Geriatric Psychiatry, 14: 1014-1018.

9. Hashimoto $S$, Sawada T, Inoue T, Yamamoto K \& Iwata M (1999). Cholinergicdrug induced sicca syndrome in Parkinson's disease: a case report and a review of the literature. Clinical Neurology and Neurosurgery, 101: 268-270.

10. Astor FC, Hanft KL \& Ciocon J O (1999). Xerostomia: a prevalent condition in the elderly. Ear, Nose and Throat J oumal, 789: 476-479.

11. Bagheri $H$, Schmidt L, Berlan $M \&$ Montastruc J L (1997). A comparative study of yohimbine and anetholtrithione on salivary secretion in depressed patients treated with psychotropic drugs. European J ournal of Clinical Pharmacology, 52: 339-342.

12. Salah RS \& Cameron OG (1996). Pilocarpine for anticholinergic adverse effects associated with desipramine treatment. American J ournal of Psychiatry, 4: 579 (Letter).

13. Lader MH (1996). Tolerability and safety: essentials in antidepressant pharmacotherapy. J ournal of Clinical Psychiatry, 57 (Suppl 2): 39-44.

14. Hunter KD \& Wilson WS (1995). The effects of antidepressant drugs on salivary flow and content of sodium and potassium ions in human parotid saliva. Archives of Oral Biology, 40: 983-989.

15. Glassman AH, Carino JS \& Roose SP (1984). Adverse effects of tricyclic antidepressants: focus on the elderly. Advances in Biochemical Psychopharmacology, 39: 391-398.

16. Bassuk E \& Schoonover S (1978). Ram- pant dental in the treatment of depression. J ournal of Clinical Psychiatry, 39: 163-165.

17. Mandel ID (1989). Preventive dental services for the elderly. Dental Clinics of North America, 33: 81-90.

18. Greenspan D \& Daniels TE (1987). Effectiveness of pilocarpine in postradiation xerostomia. Cancer, 59: 1123-1125.

19. Zimmerman RP, Mark RJ, Tran LM \& J uillard GF (1997). Concomitant pilocarpine during head and neck irradiation is associated with decreased post-treatment xerostomia. International J ournal of Radiation Oncology, Biology, Physics, 37: 571-575.

20. Brown J H \& Taylor P (1996). Muscarinic receptor agonists and antagonists. In: Gilman AG \& Goodman A (Editors), The Pharmacological Basis of Therapeutics. Chapter 7. 9th edn. McGraw-Hill Publishing, New York, NY, USA, 141-160.

21. Ferguson MM (1993). Pilocarpine and other cholinergic drugs in the management of salivary gland dysfunction. Oral Surgery, Oral Medicine and Oral Pathology, 75: 186-191.

22. Bell M, Askari A, Bookman A, Frydrych S, Lamont J, McComb J, Muscoplat C\& Slomovic A (1999). Sjögren's syndrome: a critical review of clinical management. J ournal of Rheumatology, 26: 2051-2061.

23. Nusair $S \&$ Rubinow A (1999). The use of oral pilocarpine in xerostomia and Sjögren's syndrome. Seminars in Arthritis and Rheumatism, 28: 360-367.

24. Vivino FB, Al-Hashimi I, Khan Z, LeVeque FG, Salisbury 3rd PL, Tran-J ohnson TK, Muscoplat CC, Trivedi M, Goldlust B \& Gallagher SC (1999). Pilocarpine tablets for the treatment of dry mouth and dry eye symptoms in patients with Sjögren syndrome: a randomized, placebo-controlled, fixed-dose, multicenter trial. P9201 Study Group. Archives of Internal Medicine, 159: 174-181.

25. Nagler RM \& Nagler A (1999). Pilocarpine hydrochloride relieves xerostomia in chronic graft-versus-host disease: a sialometrical study. Bone Marrow Transplantation, 23: 1007-1011.
26. Niedermeier $W, M$ atthaeus $C$, Meyer $C$, Staar S, Muller RP \& Schulze HJ (1998). Radiation-induced hyposalivation and its treatment with oral pilocarpine. Oral Surgery, Oral Medicine and Oral Pathology, 86: 541-549.

27. Cooper RA, Cowan RA, Owens SE, J eans SP, Roberts J K, Hillel PG, Slevin NJ , Allan E, Gupta NK \& Collins CD (1999). Does salivary gland scintigraphy predict response to pilocarpine in patients with post-radiotherapy xerostomia? European J ournal of Nuclear Medicine, 26: 220-225.

28. Schuller $D E$, Stevens $P$, Clausen $K P$, Olsen J , Gahbauer R \& Martin M (1989). Treatment of radiation side effects with oral pilocarpine. J ournal of Surgical Oncology, 42: 272-276.

29. Nieuw Amerongen AVN, Aarsman ME, Bos-Vregdenhil AP \& Poukema PA (1984). Influence of autonomic agonists on the in vitro incorporation of $\left[{ }^{3} \mathrm{H}\right]$ and $\mathrm{N}$-acetyl $\left[{ }^{14} \mathrm{C}\right]$ mannosamine into submandibular action of the mouse. Biochimica et Biophysica Acta, 798: 103-110.

30. O'Connel AC, Pearson SK \& Bowen WH (1994). Pilocarpine alters caries development in partially desalivated rats. J oumal of Dental Research, 73: 637-648.

31. Lach AS \& Connel R (1990). Reversal of fissure caries in the albino rat by stimulating salivary flow with pilocarpine. Caries Research, 24: 127-129.

32. Ortiz GC, Pearson JK \& Bowen WH (1992). Influence of pilocarpine, propranolol and atropine on susceptibility to infection. J ournal of Dental Research, 71: 129 (Abstract).

33. Hunt TL (1993). A double-blind, placebocontrolled, multiple-dose, tolerance and pharmacokinetic study of oral pilocarpine hydrochloride $(\mathrm{HCL})$ in healthy male subjects. MGI Pharma (Minneapolis) Data on File. MGI 647.83, 24: 91-93.

34. Hunt TL (1991). The effect of a high meal on the bioavailability of oral pilocarpine hydrochloride $(\mathrm{HCL})$ in healthy male subjects. MGI Pharma (M inneapolis) Data on File. MGI 647.83, 27: 91-92. 